\title{
An Initial Value Problem Involving Caputo-Hadamard Fractional Derivative: The Extremal Solutions and Stabilization
}

\author{
Donal O'REGAN ${ }^{1}$, Van Hoa $N G O^{2,3, *}$ \\ ${ }^{1}$ School of Mathematics, Statistics and Applied Mathematics, National University of Ireland, \\ Galway, Ireland \\ ${ }^{2}$ Division of Computational Mathematics and Engineering, Institute for Computational Science, \\ Ton Duc Thang University,Ho Chi Minh City, Vietnam \\ ${ }^{3}$ Faculty of Mathematics and Statistics, Ton Duc Thang University, Ho Chi Minh City, Vietnam \\ *Corresponding Author: Van Hoa NGO (Email: ngovanhoa@tdtu.edu.vn ) \\ (Received: 28-Feb-2020; accepted: 04-May-2020; published: 30-Jun-2020) \\ DOI: http://dx.doi.org/10.25073/jaec.202042.283
}

\begin{abstract}
In this paper, the existence of extremal solutions of Caputo-Hadamard-type fractional differential equations (CHFDEs) with order $\alpha \in(1,2)$ is established by employing the method of lower and upper solutions. Moreover, sufficient condition that ensure the stability of a class of CHFDE is also provided. Some examples are given to illustrate our main results.
\end{abstract}

\section{Keywords}

Caputo-Hadamard Fractional Derivative, Fractional Differential Equations, Stabilization of Fractional Differential Equations.

\section{Introduction}

Fractional calculus and fractional differential equation models have been studied in a variety of fields such as physics, mathematics, engineering, bioengineering, and other applied sciences. For a general overview of the theory and applications of fractional differential equations involving the Riemann-Liouville fractional derivative and the Caputo derivative, we refer the reader to the monograph of Kilbas [7]. Recently, fractional differential equations with the Hadamard derivative and the Caputo-Hadamard derivative have attracted the attention of a large number of researchers (see [1, 2, 4, 8, 13] and the references therein). In particular, Caputo-Hadamard fractional derivatives were introduced by Jarad et al. [5], and it was shown that there are many advantages over the usual Hadamard fractional derivative. Moreover, Gambo et al. [4] presented the fundamental theorem of fractional calculus in the Caputo-Hadamard setting based on the concept in [5], and recently Almeida [2] proposed three types of Caputo-Hadamard derivatives of variable fractional order, and studied the relation between them. Adjabi et al. [1] investigated the existence of solutions to fractional differential equations with the CaputoHadamard derivative using Banach's fixed point theorem. Yukunthorn et al. [13] studied the existence of solutions for impulsive hybrid systems of Caputo-Hadamard fractional differential equations equipped with integral boundary conditions using fixed point theorems.

In this work, we present some existence results for the initial value problem for fractional differential equations with order $\alpha \in(1,2)$ involving Caputo-Hadamard fractional derivative using 
the method of upper and lower solutions coupled with its associated monotone iteration scheme. This technique is a powerful tool for proving the existence of solutions of nonlinear fractional differential equations; see [3, 9, 10, 14, 15, 12]. To the best of our knowledge this technique has not been applied to the initial value problem for CHFDE, when $\alpha \in(1,2)$, to investigate the existence of minimal and maximal solutions. In this paper, using this technique we discuss the existence of extremal solutions and the uniqueness of the solution of the following fractional differential equation with the Caputo-Hadamard-type fractional derivative for $\alpha \in(1,2)$

$$
\left\{\begin{array}{l}
{ }^{C-H} D_{a^{+}}^{\alpha} \psi(t)=f(t, \psi(t)), \\
\psi(a)=\psi_{0}, \quad \psi^{\prime}(a)=\xi_{0},
\end{array}\right.
$$

where $t \in(a, b]$, and $a \geq 1$.

Section 2 contains some basic definitions and the related lemma that will be used. In Section 3 , using the method of upper and lower solutions, we prove the existence of extremal solutions of problem (1). Finally, the stabilization of a class of fractional differential equations is established in Section 4.

\section{Preliminaries}

In this section, some basic definitions, propositions, remarks and lemma are introduced (see $[5,4]$ for more detail), which will be used in the following discussions. Denote by $C[a, b]$, $A C[a, b]$ the space of continuous functions and the space of absolutely continuous functions from $[a, b]$ into $\mathbb{R}$, respectively. In this paper, we denote by $A C^{n}[a, b], C^{n}[a, b]$ and $C_{\gamma}[a, b]$, where $n \in \mathbb{N}$, the spaces defined by

$$
\begin{aligned}
& A C^{n}[a, b]:= \\
& \left\{\psi:[a, b] \rightarrow \mathbb{R}:\left(t \frac{d}{d t}\right)^{n-1} \psi(t) \in A C[a, b]\right\}, \\
& C^{n}[a, b]:= \\
& \quad\left\{\psi:[a, b] \rightarrow \mathbb{R}:\left(t \frac{d}{d t}\right)^{n} \psi(t) \in C[a, b]\right\} . \\
& C_{\gamma}[a, b]:= \\
& \left\{\psi:(a, b] \rightarrow \mathbb{R}:(\ln t-\ln a)^{\gamma} \psi(t) \in C[a, b]\right\},
\end{aligned}
$$

where $\gamma \in(0,1]$. If $n=1$, the space $A C^{1}[a, b]$ coincides with $A C[a, b]$. Now, we provide some definitions and properties of fractional calculus.

The Hadamard fractional integral of the function $\psi$ is defined by (see [7]):

$$
\begin{aligned}
\psi_{\alpha}(t): & =\left({ }^{H} I_{a^{+}}^{\alpha} \psi\right)(t) \\
& =\frac{1}{\Gamma(\alpha)} \int_{a}^{t}(\ln t-\ln s)^{\alpha-1} \psi(s) \frac{d s}{s}, t>a .
\end{aligned}
$$

The Hadamard fractional derivative of order $\alpha \in$ $(1,2)$ for the function $\psi$ is defined as follows (see [7]):

$$
\begin{aligned}
& \left({ }^{H} D_{a^{+}}^{\alpha} \psi\right)(t)=\left(t \frac{d}{d t}\right)^{2}{ }^{H} I_{a^{+}}^{2-\alpha} \psi(t) \\
& =\frac{1}{\Gamma(2-\alpha)}\left(t \frac{d}{d t}\right)^{2} \int_{a}^{t}(\ln t-\ln s)^{1-\alpha} \psi(s) \frac{d s}{s} .
\end{aligned}
$$

Let $\psi \in L^{1}[a, b]$. If $\left({ }^{H} D_{a^{+}}^{\alpha} \psi\right)(t)$ exists on $[a, b]$, the Caputo-Hadamard fractional derivative $\left({ }^{C-H} D_{a^{+}}^{\alpha} \psi\right)$ of order $\alpha \in(1,2)$ is defined by (see $[5,4])$

$$
\begin{aligned}
& \left({ }^{C-H} D_{a^{+}}^{\alpha} \psi\right)(t) \\
& ={ }^{H} D_{a^{+}}^{\alpha}\left[\psi(t)-\sum_{k=0}^{1} \frac{(\ln t-\ln a)^{k}}{k !}\left[\psi^{(k)}(t)\right]_{t=a}\right],
\end{aligned}
$$

where $\psi^{(k)}(t):=\left(t \frac{d}{d t}\right)^{k} \psi(t)$. Then, one has $($ see $[4,5])$

$$
\begin{aligned}
& \left({ }^{C-H} D_{a^{+}}^{\alpha} \psi\right)(t) \\
& ={ }^{H} D_{a^{+}}^{\alpha} \psi(t)-\sum_{k=0}^{1} \frac{\psi^{(k)}(a)}{k !}{ }^{H} D_{a^{+}}^{\alpha}(\ln t-\ln a)^{k} \\
& ={ }^{H} D_{a^{+}}^{\alpha} \psi(t)-\sum_{k=0}^{1} \frac{\psi^{(k)}(a)(\ln t-\ln a)^{k-\alpha}}{\Gamma(k-\alpha+1)} .
\end{aligned}
$$

On the other hand, the definition of the CaputoHadamard fractional derivative $\left({ }^{C-H} D_{a^{+}}^{\alpha} \psi\right)$ of order $\alpha \in(1,2)$ is defined by (see $[4,5])$

$$
\begin{aligned}
& \left({ }^{C-H} D_{a^{+}}^{\alpha} \psi\right)(t) \\
& =\frac{1}{\Gamma(2-\alpha)} \int_{a}^{t}(\ln t-\ln s)^{1-\alpha}\left(s \frac{d}{d s}\right)^{2} \psi(s) \frac{d s}{s} .
\end{aligned}
$$


Furthermore, we observe that:

$$
\begin{aligned}
\left\|\psi_{\alpha}\right\|_{0}: & =\sup _{t \in[a, b]}\left|\psi_{\alpha}(t)\right| \\
& \leq\|\psi\|_{0} \frac{1}{\Gamma(\alpha)} \int_{a}^{t}(\ln t-\ln s)^{\alpha-1} \frac{d s}{s} \\
& \leq \frac{\|\psi\|_{0}}{\Gamma(\alpha+1)}(\ln b-\ln a)^{\alpha},
\end{aligned}
$$

where $t \in[a, b]$ and $\|\psi\|_{0}=\sup _{t \in[a, b]}|\psi(t)|$.

Example 1. We consider $\psi(t)=(\ln t-\ln a)^{\beta}$, where $t \in[a, b]$ and $\beta \geq 2$. For $\alpha \in(1,2)$ one has that

$$
\begin{aligned}
& \left({ }^{C-H} D_{a^{+}}^{\alpha} \psi\right)(t)={ }^{H} D_{a^{+}}^{\alpha}(\ln t-\ln a)^{\beta} \\
& -\left[\begin{array}{c}
(\psi(a)) \frac{1}{\Gamma(1-\alpha)}(\ln t-\ln a)^{-\alpha} \\
+\left(a \psi^{\prime}(a) \frac{1}{\Gamma(2-\alpha)}(\ln t-\ln a)^{1-\alpha}\right.
\end{array}\right] \\
& =\frac{\Gamma(\beta+1)}{\Gamma(\beta+1-\alpha)}(\ln t-\ln a)^{\beta-\alpha} .
\end{aligned}
$$

Also one has

$$
\begin{aligned}
& \left({ }^{C-H} D_{a^{+}}^{\alpha} \psi\right)(t) \\
& =\int_{a}^{t} \frac{(\ln t-\ln s)^{1-\alpha}}{\Gamma(2-\alpha)}\left[\left(s \frac{d}{d s}\right)^{2}(\ln s-\ln a)^{\beta}\right] \frac{d s}{s} \\
& =\frac{\beta(\beta-1)}{\Gamma(2-\alpha)} \int_{a}^{t}(\ln t-\ln s)^{1-\alpha}(\ln s-\ln a)^{\beta-2} \frac{d s}{s} \\
& =B(2-\alpha, \beta-1) \frac{\beta(\beta-1)}{\Gamma(2-\alpha)}(\ln t-\ln a)^{\beta-\alpha} \\
& =\frac{\Gamma(\beta+1)}{\Gamma(\beta+1-\alpha)}(\ln t-\ln a)^{\beta-\alpha},
\end{aligned}
$$

where we make the substitution $z=\frac{\ln s-\ln a}{\ln t-\ln a}$ and use the definition of the Beta function.

Remark 1. (see [7]) Let $\alpha, \beta>0$ and $\psi, \xi \in$ $L^{p}[a, b](1 \leq p \leq \infty)$. We have that: (i) ${ }^{H} I_{a^{+}}^{\alpha}(\psi+\bar{\xi})(t)={ }^{H} I_{a^{+}}^{\alpha} \psi(t)+{ }^{H} I_{a^{+}}^{\alpha} \xi(t)$; ${ }^{H} I_{a^{+}}^{\alpha}{ }^{H} I_{a^{+}}^{\beta} \psi(t)={ }^{H} I_{a^{+}}^{\alpha+\beta} \psi(t)$.

Proposition 2. .1. (see [7]) Let $\psi \in L^{p}[a, b]$, then for $0<\alpha<\beta$ we have

$$
\left({ }^{H} D_{a^{+}}^{\alpha}{ }^{H} I_{a^{+}}^{\alpha} \psi\right)(t)=\psi(t),
$$

and

$$
\left({ }^{H} D_{a^{+}}^{\alpha}{ }^{H} I_{a^{+}}^{\beta} \psi\right)(t)=\left({ }^{H} I_{a^{+}}^{\beta-\alpha} \psi\right)(t) .
$$

Remark 2. (see [7]) Let $\psi \in L^{1}[a, b]$ such that $\psi_{1-\alpha} \in A C^{2}[a, b]$. Then we have

$$
\begin{aligned}
& { }^{H} I_{a^{+}}^{\alpha}{ }^{H} D_{a^{+}}^{\alpha} \psi(t) \\
& =\psi(t)-\sum_{k=1}^{2} \frac{\psi_{(2-\alpha)}^{(2-k)}(a)}{\Gamma(\alpha-k+1)}(\ln t-\ln a)^{\alpha-k}
\end{aligned}
$$

for $t \in(a, b]$.

Remark 3. (see $[4,5]$ ) If $\psi \in A C^{2}[a, b]$ or $C^{2}[a, b]$, then

$$
\begin{aligned}
& \left({ }^{H} I_{a^{+}}^{\alpha}{ }^{C-H} D_{a^{+}}^{\alpha} \psi\right)(t) \\
& =\psi(t)-\sum_{k=0}^{1} \frac{\psi^{(k)}(a)}{k !}(\ln t-\ln a)^{k},
\end{aligned}
$$

where $t \in(a, b]$, and

$$
\left({ }^{C-H} D_{a^{+}}^{\alpha H} I_{a^{+}}^{\alpha} \psi\right)(t)=\psi(t), \quad t \in(a, b] .
$$

Example 2. Let $\alpha \in(1,2)$ and $\psi(t)=(\ln t-$ $\ln a)^{2}$, where $t \in[a, b]$. One can see that the right-hand sides of (8) and (9) are equal $(\ln t-$ $\ln a)^{2}$. Also it is well-known that (see page 112 in [7])

$$
{ }^{H} I_{a^{+}}^{\alpha}(\ln t-\ln a)^{\beta-1}=\frac{\Gamma(\beta)(\ln t-\ln a)^{\beta+\alpha-1}}{\Gamma(\beta+\alpha)},
$$

where $\beta>0$. Then, from Example 1 we get

$$
\left({ }^{H} I_{a^{+}}^{\alpha}{ }^{C-H} D_{a^{+}}^{\alpha} \psi\right)(t)=(\ln t-\ln a)^{2},
$$

and

$$
\left({ }^{C-H} D_{a^{+}}^{\alpha H} I_{a^{+}}^{\alpha} \psi\right)(t)=(\ln t-\ln a)^{2} .
$$

Lemma 1. [6] Let $\mathbb{X}$ be an ordered Banach space, $u_{0}, v_{0} \in \mathbb{X}, u_{0} \leq v_{0}, \mathbb{D}=\left[u_{0}, v_{0}\right], \mathbb{P}$ : $\mathbb{D} \rightarrow \mathbb{X}$ be an increasing completely continuous operator and $u_{0} \leq \mathbb{P} u_{0}, v_{0} \geq \mathbb{P} v_{0}$. Then, the operator $\mathbb{P}$ has a minimal fixed point $u^{*}$ and a maximal fixed point $v^{*}$. Furthermore, if we let $u_{n}=\mathbb{P} u_{n-1}, v_{n}=\mathbb{P} v_{n-1}, n=1,2,3, \ldots$, then $u_{0} \leq u_{1} \leq u_{2} \leq \ldots \leq u_{n} \leq \ldots \leq v_{n} \leq \ldots \leq v_{2} \leq$ $v_{1} \leq v_{0}$, and $u_{n} \rightarrow u^{*}, v_{n} \rightarrow v^{*}$. 


\section{The existence of extremal solutions}

Consider the following fractional differential equation with the order $\alpha \in(1,2)$ :

$$
\left\{\begin{array}{l}
{ }^{C-H} D_{a^{+}}^{\alpha} \psi(t)=f(t, \psi(t)), \\
\psi(a)=\psi_{0}, \quad \psi^{\prime}(a)=\xi_{0}
\end{array}\right.
$$

where $t \in(a, b]$, and $a \geq 1$. A function $\psi$ : $[a, b] \rightarrow \mathbb{R}$ is said to be a solution of the problem (10) if it satisfies $\psi(a)=\psi_{0}, \psi^{\prime}(a)=\xi_{0}$ and ${ }^{C-H} D_{a^{+}}^{\alpha} \psi(t)=f(t, \psi), t \in(a, b]$.

Lemma 2. Let $f:(a, b] \times \mathbb{R} \rightarrow \mathbb{R}$ be such that $t \mapsto f(t, u)$ belongs to $C_{\gamma}([a, b], \mathbb{R}), 0 \leq \gamma \leq 1$. Then, a function $\psi$ is a solution of the problem (10) if and only if $\psi$ satisfies

$$
\begin{aligned}
& \psi(t)=\psi_{0}+\psi^{(1)}(a)(\ln t-\ln a) \\
& +\frac{1}{\Gamma(\alpha)} \int_{a}^{t}(\ln t-\ln s)^{\alpha-1} f(s, \psi(s)) \frac{d s}{s} .
\end{aligned}
$$

Proof. Let $\psi \in C[a, b]$ be a solution of the problem (10). Then by (10) and Remark 3 one has that

$$
\begin{aligned}
& \left({ }^{H} I_{a^{+}}^{\alpha}{ }^{C-H} D_{a^{+}}^{\alpha} \psi\right)(t) \\
& =\psi(t)-\psi(a)-\psi^{(1)}(a)(\ln t-\ln a),
\end{aligned}
$$

Because $f(t, u) \in C_{\gamma}([a, b], \mathbb{R})$ and from problem (10), we have that

$$
\begin{aligned}
& \left({ }^{H} I_{a^{+}}^{\alpha}{ }^{C-H} D_{a^{+}}^{\alpha} \psi\right)(t)={ }^{H} I_{a^{+}}^{\alpha} f(t, \psi(t)) \\
& =\frac{1}{\Gamma(\alpha)} \int_{a}^{t}(\ln t-\ln s)^{\alpha-1} f(s, \psi(s)) \frac{d s}{s},
\end{aligned}
$$

This yields the necessity condition of the proof. On the other hand, because of the continuity of the function $f$, the function $t \mapsto$ $f_{\alpha}(t, u)$ is continuous on $(a, b]$ and $f_{\alpha}(a, u(a))=$ $\lim _{t \rightarrow a^{+}} f_{\alpha}(t, u(t))=0$. Then, $\psi(a)=\psi_{0}$ and $\psi^{\prime}(a)=\xi_{0}$. By taking the operator ${ }^{H} D_{a^{+}}^{\alpha}$ on two sides of (11) and by Proposition 2. .1, one gets

$$
\begin{aligned}
& { }^{H} D_{a^{+}}^{\alpha}\left[\psi(\cdot)-\psi(a)-\psi^{(1)}(a)(\ln t-\ln a)\right](t) \\
& =f(t, \psi(t)) .
\end{aligned}
$$

To show the main results of this paper, we need the formula of solution of the problem (10) in the linear form as the below.

Remark 4. The formula of the solution of the following linear Caputo-Hadamard fractional differential equation

$$
\left\{\begin{array}{l}
{ }^{C-H} D_{a^{+}}^{\alpha} \psi(t)=\lambda \psi(t)+h(t), \\
\psi(a)=\psi_{0}, \quad \psi^{\prime}(a)=\xi_{0}
\end{array}\right.
$$

is expressed by

$$
\begin{aligned}
& \psi(t)=\psi_{0} E_{\alpha, 1}\left(\lambda(\ln t-\ln a)^{\alpha}\right) \\
& +\psi^{(1)}(a)(\ln t-\ln a) E_{\alpha, 2}\left(\lambda(\ln t-\ln a)^{\alpha}\right) \\
& +\int_{a}^{t}(\ln t-\ln s)^{\alpha-1} E_{\alpha, \alpha}\left(\lambda(\ln t-\ln s)^{\alpha}\right) h(s) \frac{d s}{s} .
\end{aligned}
$$

Indeed, to get the explicit formula of the solution of (14), we shall employ the method of successive approximations. First of all, based on Lemma 2 we observe that a function $\psi$ is a solution of the problem ( 14) if it satisfies

$$
\begin{aligned}
\psi(t) & =\psi_{0}+\psi^{(1)}(a)(\ln t-\ln a) \\
& +\lambda\left({ }^{H} I_{a^{+}}^{\alpha} \psi\right)(t)+\left({ }^{H} I_{a^{+}}^{\alpha} h\right)(t) .
\end{aligned}
$$

Next, we set $\psi_{0}(t)=\psi_{0}+\psi^{(1)}(a)(\ln t-\ln a)$ and for $n=1,2,3, \ldots$

$$
\psi_{n}(t)=\psi_{0}(t)+\lambda\left({ }^{H} I_{a^{+}}^{\alpha} \psi_{n-1}\right)(t)+\left({ }^{H} I_{a^{+}}^{\alpha} h\right)(t) .
$$

For $n=1$, because ${ }^{H} I_{a^{+}}^{\alpha}(\ln t-\ln a)^{\beta}=$ $\frac{\Gamma(\beta+1)}{\Gamma(\alpha+\beta+1)}(\ln t-\ln a)^{\alpha+\beta}$, where $\beta \geq 0$, we have that

$\psi_{1}(t)=\psi_{0}(t)+\lambda\left({ }^{H} I_{a^{+}}^{\alpha}\left[\psi_{0}+\psi^{(1)}(a)(\ln t-\ln a)\right]\right.$ $+\left({ }^{H} I_{a^{+}}^{\alpha} h\right)(t)$

$=\psi_{0}+\psi^{(1)}(a)(\ln t-\ln a)+\lambda \frac{(\ln t-\ln a)^{\alpha}}{\Gamma(\alpha+1)} \psi_{0}$

$+\lambda \psi^{(1)}(a) \Gamma(2) \frac{(\ln t-\ln a)^{\alpha+1}}{\Gamma(\alpha+2)}+\left({ }^{H} I_{a^{+}}^{\alpha} h\right)(t)$

$=\psi_{0}\left[1+\frac{\lambda(\ln t-\ln a)^{\alpha}}{\Gamma(\alpha+1)}\right]$

$+\psi^{(1)}(a)(\ln t-\ln a)\left[1+\frac{\lambda(\ln t-\ln a)^{\alpha} \Gamma(2)}{\Gamma(\alpha+2)}\right]$

$+\left({ }^{H} I_{a^{+}}^{\alpha} h\right)(t)$. 
For $n=2$, we also see that

$$
\begin{aligned}
\psi_{2}(t) & =\psi_{0}\left[\begin{array}{c}
1+\frac{\lambda(\ln t-\ln a)^{\alpha}}{\Gamma(\alpha+1)} \\
+\frac{\lambda^{2}(\ln t-\ln a)^{2 \alpha}}{\Gamma(2 \alpha+1)}
\end{array}\right] \\
& +\left({ }^{H} I_{a^{+}}^{\alpha} h\right)(t)+\lambda\left({ }^{H} I_{a^{+}}^{2 \alpha} h\right)(t) \\
& +\psi^{(1)}(a)(\ln t-\ln a)\left[\begin{array}{c}
1+\frac{\lambda(\ln t-\ln a)^{\alpha}}{\Gamma(\alpha+2)} \\
+\frac{\lambda^{2}(\ln t-\ln a)^{2 \alpha}}{\Gamma(2 \alpha+2)}
\end{array}\right] .
\end{aligned}
$$

If one proceeds inductively and let $n \rightarrow \infty$, one gets the solution

$$
\begin{aligned}
& \psi(t)=\psi_{0} \sum_{i=0}^{\infty} \frac{\lambda^{i}(\ln t-\ln a)^{i \alpha}}{\Gamma(i \alpha+1)} \\
& +\psi^{(1)}(a)(\ln t-\ln a) \sum_{i=0}^{\infty} \frac{\lambda^{i}(\ln t-\ln a)^{i \alpha}}{\Gamma(i \alpha+2)} \\
& +\int_{a}^{t} \sum_{i=0}^{\infty} \frac{\lambda^{i}(\ln t-\ln s)^{i \alpha+(\alpha-1)}}{\Gamma(i \alpha+\alpha)} h(s) \frac{d s}{s} \\
& =\psi_{0} \sum_{i=0}^{\infty} \frac{\lambda^{i}(\ln t-\ln a)^{i \alpha}}{\Gamma(i \alpha+1)} \\
& +\psi^{(1)}(a)(\ln t-\ln a) \sum_{i=0}^{\infty} \frac{\lambda^{i}(\ln t-\ln a)^{i \alpha}}{\Gamma(i \alpha+2)} \\
& +\int_{a}^{t}(\ln t-\ln s)^{\alpha-1} \sum_{i=0}^{\infty} \frac{\lambda^{i}(\ln t-\ln s)^{i \alpha}}{\Gamma(i \alpha+\alpha)} h(s) \frac{d s}{s} .
\end{aligned}
$$

Then, by using the definition of the MittagLeffler function $E_{\alpha, \beta}(u)=\sum_{i=0}^{\infty} \frac{u^{i}}{\Gamma(i \alpha+\beta)}, \alpha, \beta>$ 0 , the solution of the problem (14) is given (15).

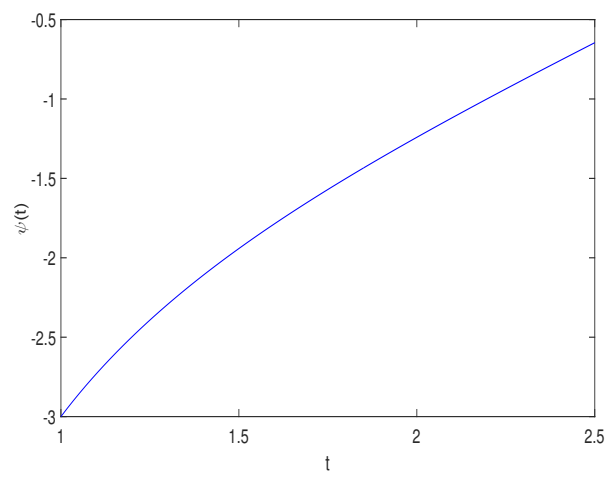

Fig. 1: The graph of $\psi(t)$ in Example 3 with $\lambda=$ $0.5, \alpha=1.75, \beta=2$.
Example 3. In Remark 4, we consider $[a, b]=$ $[1, e]$, the function $h(t)=2(\ln t)^{\beta}, \psi(1)=$ $-3, \psi^{\prime}(1)=3$. Then, we get the formula of the solution as follows:

$$
\begin{aligned}
& \psi(t)=-3 E_{\alpha, 1}\left(\lambda(\ln t)^{\alpha}\right)+3 \ln t E_{\alpha, 2}\left(\lambda(\ln t)^{\alpha}\right) \\
& +2 \int_{1}^{t}(\ln t-\ln s)^{\alpha-1} E_{\alpha, \alpha}\left(\lambda(\ln (t / s))^{\alpha}\right) \ln ^{\beta} s \frac{d s}{s} \\
& =-3 E_{\alpha, 1}\left(\lambda(\ln t)^{\alpha}\right)+3 \ln t E_{\alpha, 2}\left(\lambda(\ln t)^{\alpha}\right) \\
& +2 \Gamma(\beta+1)(\ln t)^{\alpha+\beta} E_{\alpha, \alpha+\beta+1}\left(\lambda(\ln t)^{\alpha}\right) .
\end{aligned}
$$

The graph of the solution $\psi(t)$ is shown in Fig. 1.

Definition 1. Let $\alpha \in(1,2)$. A function $\psi^{L} \in$ $C_{2-\alpha}[a, b]$ is a lower solution for the initial value problem (10) if

$$
\left\{\begin{array}{l}
{ }^{C-H} D_{a^{+}}^{\alpha} \psi^{L}(t) \leq f\left(t, \psi^{L}(t)\right) \\
\psi^{L}(a) \leq \psi_{0}, \quad\left(\psi^{L}\right)^{\prime}(a) \leq \xi_{0}
\end{array}\right.
$$

A function $\psi^{U} \in C_{2-\alpha}[a, b]$ is an upper solution for (10) if it satisfies the reverse inequalities of (16), i.e.,

$$
\left\{\begin{array}{l}
{ }^{C-H} D_{a^{+}}^{\alpha} \psi^{U}(t) \geq f\left(t, \psi^{U}(t)\right) \\
\psi^{U}(a) \geq \psi_{0}, \quad\left(\psi^{U}\right)^{\prime}(a) \geq \xi_{0}
\end{array}\right.
$$

As in the proof of Lemma 2.2 in [11], we also get the remark below.

Remark 5. Let $\alpha \in(1,2), \beta \in[1,2], b<\infty$ and $\lambda<0$. Then we have that

$$
\sum_{i=1}^{\infty} \frac{i\left[\lambda(\ln t-\ln a)^{\alpha}\right]^{i-1}}{\Gamma(i \alpha+\beta)}>0, \quad \forall t \in[a, b] .
$$

Remark 6. Let $\alpha \in(1,2)$ and $\lambda<0$. We observe that the Mittag-Leffler functions in Remark 4 satisfies $E_{\alpha, \alpha}(0)=1 / \Gamma(\alpha), E_{\alpha, 1}(0)=$ $1, E_{\alpha, 2}(0)=1$. In addition, the following properties are satisfied:

(i) for all $t, s \in[a, b]$, where $1 \leq a \leq s<t \leq b$,

$$
\begin{aligned}
& \left|E_{\alpha, 1}\left(\lambda(\ln t-\ln a)^{\alpha}\right)\right| \leq 1, \\
& \left|E_{\alpha, 2}\left(\lambda(\ln t-\ln a)^{\alpha}\right)\right| \leq 1, \\
& \left|E_{\alpha, \alpha}\left(\lambda(\ln t-\ln s)^{\alpha}\right)\right| \leq \frac{1}{\Gamma(\alpha)} .
\end{aligned}
$$


(ii) for any $t_{1}, t_{2} \in[a, b]$ and $t_{1} \leq t_{2}$, where we have that $a \geq 1$,

$$
\begin{aligned}
E_{\alpha, 1} & \left(\lambda\left(\ln t_{2}-\ln a\right)^{\alpha}\right) \\
\leq & E_{\alpha, 1}\left(\lambda\left(\ln t_{1}-\ln a\right)^{\alpha}\right), \\
E_{\alpha, 2}( & \left.\lambda\left(\ln t_{2}-\ln a\right)^{\alpha}\right) \\
\leq & E_{\alpha, 2}\left(\lambda\left(\ln t_{1}-\ln a\right)^{\alpha}\right), \\
E_{\alpha, \alpha} & \left(\lambda\left(\ln t_{2}-\ln a\right)^{\alpha}\right) \\
\leq & E_{\alpha, \alpha}\left(\lambda\left(\ln t_{1}-\ln a\right)^{\alpha}\right) .
\end{aligned}
$$

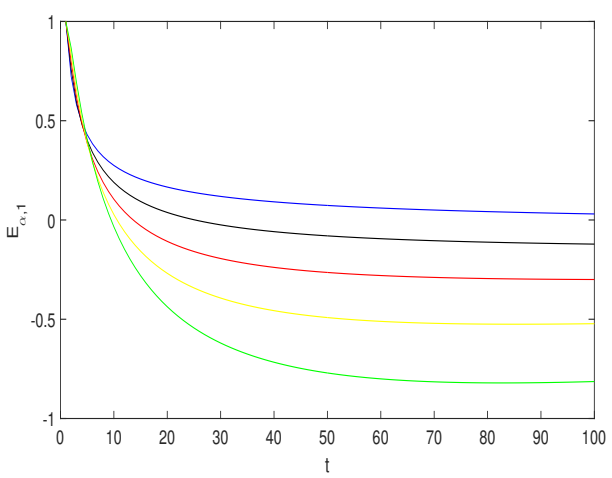

Fig. 2: The graph of $E_{\alpha, 1}\left(\lambda(\ln t-\ln a)^{\alpha}\right)$ with $\lambda=-0.5, \alpha=1.1$ (blue), $\alpha=$ 1.3 (black), $\alpha=1.5$ (red), $\alpha=1.7$ (yellow), and $\alpha=1.9$ (green).

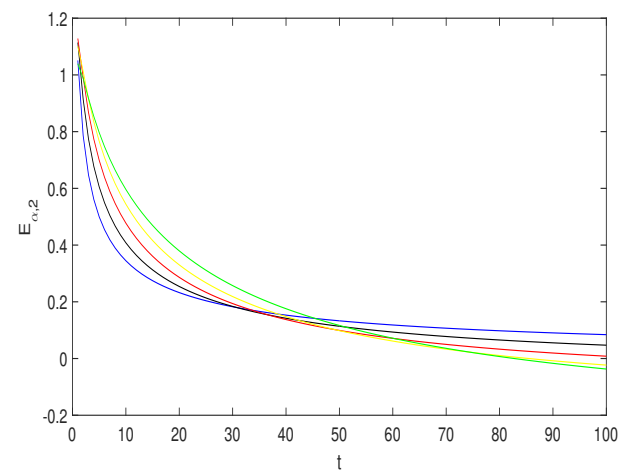

Fig. 3: The graph of $E_{\alpha, 2}\left(\lambda(\ln t-\ln a)^{\alpha}\right)$ with $\lambda=-0.5, \alpha=1.1$ (blue), $\alpha=$ 1.3 (black), $\alpha=1.5$ (red), $\alpha=1.7$ (yellow), and $\alpha=1.9$ (green).

Proof. Based on the definition of the MittagLeffler function $E_{\alpha, \beta}\left(\lambda(\ln t-\ln a)^{\alpha}\right)=$ $\sum_{i=0}^{\infty} \frac{\lambda^{i}(\ln t-\ln a)^{i \alpha}}{\Gamma(i \alpha+\beta)}$, where $\alpha \in(1,2), \beta \in$ $\{1,2, \alpha\}$, and by passing to limit with $t \rightarrow a^{+}$,

$$
\lim _{t \rightarrow a^{+}} E_{\alpha, \beta}\left(\lambda(\ln t-\ln a)^{\alpha}\right)=\frac{1}{\Gamma(\beta)} .
$$

This yields that $E_{\alpha, \alpha}(0)=1 / \Gamma(\alpha), E_{\alpha, 1}(0)=$ $1, E_{\alpha, 2}(0)=1$. Next, to show assertions (i) and (ii) we shall prove that the Mittag-Leffler functions $E_{\alpha, \alpha}, E_{\alpha, 1}, E_{\alpha, 2}$ are decreasing in $t \in[a, b]$. Let $\alpha \in(1,2)$ and $\beta \in\{1,2, \alpha\}$, and then we make the direct calculation

$$
\begin{aligned}
& \frac{d}{d t} E_{\alpha, \beta}\left(\lambda(\ln t-\ln a)^{\alpha}\right) \\
& =\frac{\lambda \alpha(\ln t-\ln a)^{\alpha-1}}{t} \sum_{i=1}^{\infty} \frac{i\left[\lambda(\ln t-\ln a)^{\alpha}\right]^{i-1}}{\Gamma(i \alpha+\beta)} .
\end{aligned}
$$

Based on Remark 5 and by $\lambda<0, \alpha \in(1,2)$, we conclude that the right-hand side of $(25)$ is negative. Thus, the function $E_{\alpha, \beta}$ is decreasing in $t$. This yields that the assertions (i) and (ii) are satisfied. From Figs. 2-4, it follows that the assertions (19)-(21) are valid. In addition, the graphs $5-7$ are given to illustrate the assertions (22)-(24).

Theorem 1. Let $\alpha \in(1,2)$ and $f$ be continuous. If the function $f$ satisfies the condition

$$
|f(t, u)-f(t, v)| \leq L|u-v|
$$

where $u, v \in \mathbb{R}$ and $L$ is a positive constant, then the initial value problem (10) has a solution $\psi \in$ $C[a, b]$. Furthermore, let $\psi^{L}, \psi^{U}$ be lower and upper solutions of (10) such that $\psi^{L}(t) \leq \psi^{U}(t)$ on $[a, b]$ and suppose further that

$$
f(t, u)-f(t, v) \geq-M(u-v)
$$

for $\psi^{L}(t) \leq v \leq u \leq \psi^{U}(t), M \geq 0$. Then there exist monotone sequences $\left\{u_{n}\right\},\left\{v_{n}\right\}$ such that $u_{n} \rightarrow \psi_{\min }, v_{n} \rightarrow \psi_{\max }$ as $n \rightarrow \infty$ uniformly and monotonically on $[a, b]$, and $\left(\psi_{\min }, \psi_{\max }\right)$ are minimal and maximal solutions of the problem (10), respectively.

Proof. To prove this theorem, let us define the following integral operator $\mathbb{P}: C[a, b] \rightarrow C[a, b]$ 


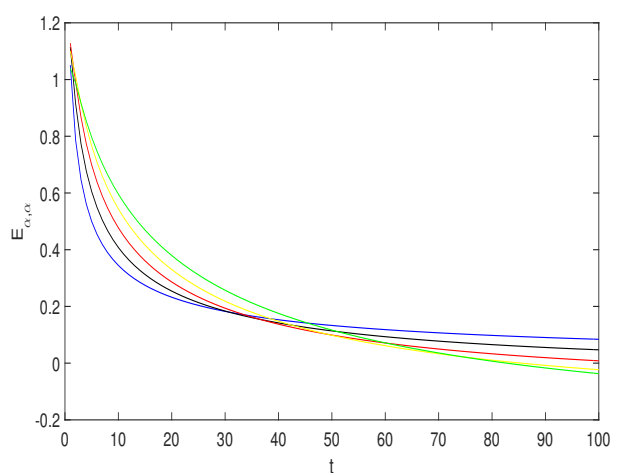

Fig. 4: The graph of $E_{\alpha, \alpha}\left(\lambda(\ln t-\ln a)^{\alpha}\right)$ with $\lambda=-0.5, \alpha=1.1$ (blue), $\alpha=$ 1.3 (black), $\alpha=1.5$ (red), $\alpha=1.7$ (yellow), and $\alpha=1.9$ (green).

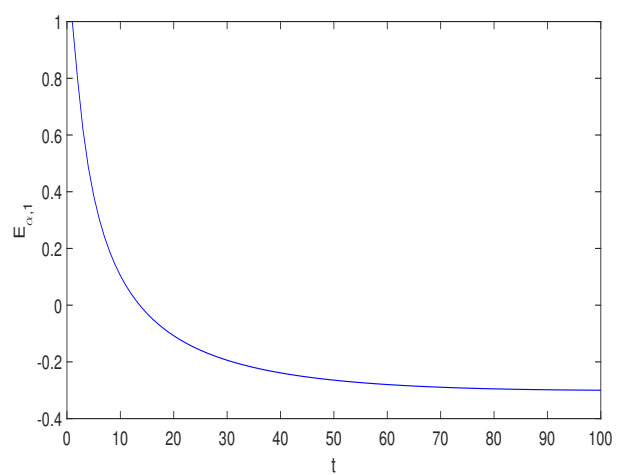

Fig. 5: The graph of $E_{1.5,1}\left(\lambda(\ln t-\ln a)^{1.5}\right)$ with $\lambda=-0.5$.

by

$$
\begin{aligned}
& (\mathbb{P} \psi)(t)=\psi_{0} E_{\alpha, 1}\left(\lambda(\ln t-\ln a)^{\alpha}\right) \\
& +\psi^{(1)}(a)(\ln t-\ln a) E_{\alpha, 2}\left(\lambda(\ln t-\ln a)^{\alpha}\right) \\
& +\int_{a}^{t} E_{\alpha, \alpha}\left(\lambda(\ln (t / s))^{\alpha}\right) \frac{[f(s, \psi(s))-\lambda \psi(s)]}{(\ln t-\ln s)^{1-\alpha}} \frac{d s}{s} .
\end{aligned}
$$

This proof consists of three steps.

Step 1: The initial value problem (10) has at least one solution if and only if the operator $\mathbb{P}$ has a fixed point $\psi$ satisfying

$$
\left\{\begin{array}{l}
{ }^{C-H} D_{a^{+}}^{\alpha} \psi(t)-\lambda \psi(t)=f(t, \psi(t))-\lambda \psi(t), \\
\psi(a)=\psi_{0}, \quad \psi^{\prime}(a)=\xi_{0},
\end{array}\right.
$$

We now show that the integral operator $\mathbb{P}$ is welldefined, that is, $\mathbb{P} \psi \in C[a, b]$ for $\psi \in C[a, b]$.

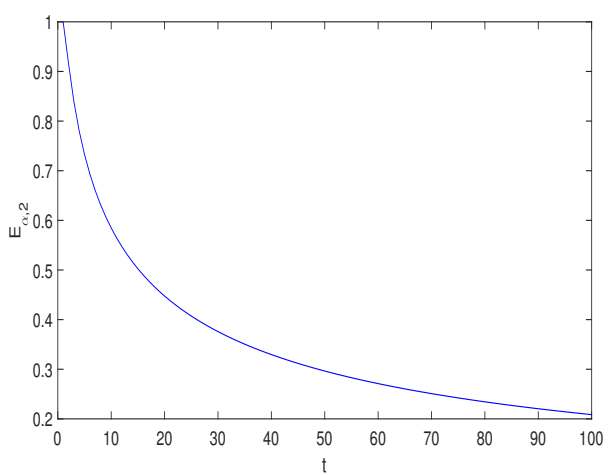

Fig. 6: The graph of $E_{1.5,2}\left(\lambda(\ln t-\ln a)^{1.5}\right)$ with $\lambda=-0.5$.

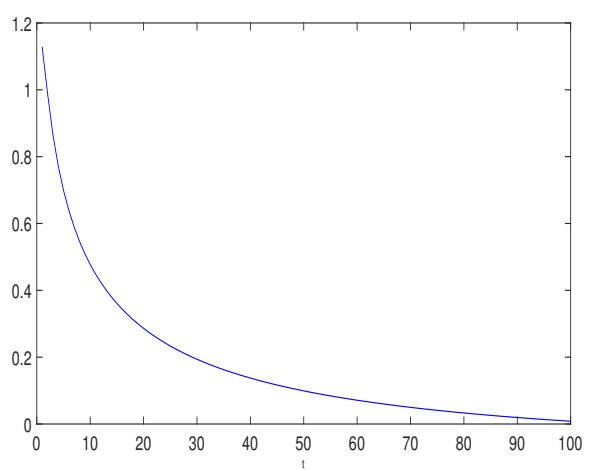

Fig. 7: The graph of $E_{1.5,1.5}\left(\lambda(\ln t-\ln a)^{1.5}\right)$ with $\lambda=-0.5$.

Let us consider $\psi_{n}, \psi \in C[a, b]$, where $\psi_{0}(t)=$ $\psi_{0}+\psi^{(1)}(a)(\ln t-\ln a)$, such that $\psi_{n} \rightarrow \psi$ as $n \rightarrow \infty$, and then from Remark 6 and hypothesis (26) we have that for $n \in \mathbb{N}$

$$
\begin{aligned}
& \left\|\mathbb{P} \psi_{n}-\mathbb{P} \psi\right\|_{0}=\max _{t \in[a, b]}\left|\left(\mathbb{P} \psi_{n}(t)-\mathbb{P} \psi(t)\right)\right| \\
& \leq \max _{t \in[a, b]} \int_{a}^{t} \frac{(\ln t-\ln s)^{\alpha-1}}{\Gamma(\alpha)}\left[\begin{array}{l}
L\left|\psi_{n}-\psi\right| \\
+|\lambda|\left|\psi_{n}-\psi\right|
\end{array}\right] \frac{d s}{s} \\
& \leq \frac{(\ln t-\ln a)^{\alpha}}{\Gamma(\alpha+1)}(L+|\lambda|)\left\|\psi_{n}-\psi\right\|_{0} .
\end{aligned}
$$

Therefore, this yields

$$
\begin{aligned}
\left\|\mathbb{P} \psi_{n}-\mathbb{P} \psi\right\|_{0} & \leq \frac{(\ln b-\ln a)^{\alpha}}{\Gamma(\alpha+1)}(L+|\lambda|)\left\|\psi_{n}-\psi\right\|_{0} \\
& \longrightarrow 0, \quad \text { as } n \rightarrow \infty .
\end{aligned}
$$

This allows to conclude that the operator $\mathbb{P}$ is continuous on $[a, b]$. Therefore, $\mathbb{P}$ is well-defined. 
Step 2: We now show that the operator $\mathbb{P}$ has a fixed point, and this is done using Schauder's fixed point theorem. In the previous step, we have $\mathbb{P} \psi \in C[a, b]$ if $\psi \in C[a, b]$, i.e. $\mathbb{P}$ maps the set $C[a, b]$ into itself. Next, let $\mathbb{S} \subset C[a, b]$ be a bounded set, and then we shall show that $\mathbb{P}(\mathbb{S})=\{(\mathbb{P} \psi)(t): \psi \in \mathbb{S}\}$ is a relatively compact set, and this is done using the Arzela-Ascoli Theorem (see Theorem 1.8 in [7]). First of all we shall verify that the set $\mathbb{P}(\mathbb{S})$ is uniformly bounded. Let $W(t) \in \mathbb{P}(\mathbb{S})$. Then from Remark 6 and the condition (26) we have that for all $t \in[a, b], \alpha \in(1,2)$,

$$
\begin{aligned}
& \|W(t)\|_{0}=\|\mathbb{P} \psi\|_{0} \leq\left|\psi_{0}\right|+\left|\psi^{(1)}(a)\right|(\ln b-\ln a) \\
& +\max _{t \in[a, b]} \int_{a}^{t} \frac{(\ln t-\ln s)^{\alpha-1}}{\Gamma(\alpha)}\left(\begin{array}{c}
L|\psi(s)| \\
+|f(t, 0)| \\
+|\lambda||\psi(s)|
\end{array}\right) \frac{d s}{s} \\
& \leq\left|\psi_{0}\right|+\left|\psi^{(1)}(a)\right|(\ln b-\ln a) \\
& +\frac{M_{f}}{\Gamma(\alpha+1)}(\ln b-\ln a)^{\alpha} \\
& +\frac{(\ln b-\ln a)^{\alpha}}{\Gamma(\alpha+1)}(L+|\lambda|)\|\psi\|_{0},
\end{aligned}
$$

where $M_{f}=\max _{t \in[a, b]}|f(t, 0)|$. This argument shows that $\mathbb{P}(\mathbb{S})$ is uniformly bounded. Next, we show that $\mathbb{P}(\mathbb{S})$ is equicontinuous. For every $\psi \in C[a, b]$, from the continuity of the function $f$, from Remark 6 and by letting $K_{f}=$ $\sup _{t \in[a, b]}|f(t, \psi)+\lambda \psi|$, we get for $a \leq t_{1} \leq t_{2} \leq$ $b$,

$$
\begin{aligned}
& \left|(\mathbb{P} \psi)\left(t_{2}\right)-(\mathbb{P} \psi)\left(t_{1}\right)\right| \leq\left|\psi_{0}\right|\left|\mathbb{E}_{1}\left(t_{2}\right)-\mathbb{E}_{1}\left(t_{1}\right)\right| \\
& +\left|\psi^{(1)}(a)\right|\left|\mathbb{E}_{2}\left(t_{2}\right)-\mathbb{E}_{2}\left(t_{1}\right)\right| \\
& +\frac{K_{f}}{\Gamma(\alpha)} \int_{a}^{t_{1}}\left[\left(\ln t_{2}-s\right)^{\alpha-1}-\left(\ln t_{1}-\ln s\right)^{\alpha-1}\right] \frac{d s}{s} \\
& +\frac{K_{f}}{\Gamma(\alpha)} \int_{t_{1}}^{t_{2}}\left(\ln t_{2}-\ln s\right)^{\alpha-1} \frac{d s}{s} \\
& =\left|\psi_{0}\right|\left|\mathbb{E}_{1}\left(t_{2}\right)-\mathbb{E}_{1}\left(t_{1}\right)\right| \\
& +\left|\psi^{(1)}(a)\right|\left|\mathbb{E}_{2}\left(t_{2}\right)-\mathbb{E}_{2}\left(t_{1}\right)\right| \\
& +\frac{K_{f}}{\Gamma(\alpha+1)}\left(\left(\ln t_{2}-\ln a\right)^{\alpha}-\left(\ln t_{1}-\ln a\right)^{\alpha}\right) \\
& +\frac{K_{f}}{\Gamma(\alpha+1)}\left(\ln t_{2}-\ln t_{1}\right)^{\alpha},
\end{aligned}
$$

where $\quad \mathbb{E}_{1}(t) \quad:=\quad E_{\alpha, 1}\left(\lambda(\ln t-\ln a)^{\alpha}\right)$, $\mathbb{E}_{2}(t):=(\ln t-\ln a) E_{\alpha, 2}\left(\lambda(\ln t-\ln a)^{\alpha}\right)$.
Since $\alpha \in(1,2), \lambda<0$, the functions $\mathbb{E}_{i}(t), i=\{1,2\}$ are uniformly continuous and bounded on $[a, b]$. Therefore, as $t_{2} \rightarrow t_{1}$, the right-hand side of the above inequality tends to zero. Thus $\mathbb{P}(\mathbb{S})$ is equicontinuous. So, by Schauder's fixed point theorem (see Theorem 1.7 in [7]), we assert that $\mathbb{P}$ has at least one fixed point $\psi^{*}$ such that $\mathbb{P} \psi^{*}=\psi^{*}$. This fixed point is the required solution of the initial value problem (10).

Step 3: In order to prove that the operator $\mathbb{P}$ has a extremal solutions, we show that the conditions of Lemma 1 are satisfied. Indeed, let $\psi^{L}, \psi^{U}$ be lower and upper solutions of the initial value problem (10). Then by the definition of the lower solution, there exist $g(t) \geq 0$ and $\varepsilon_{1}, \varepsilon_{2} \geq 0$ such that

$$
\left\{\begin{array}{l}
{ }^{C-H} D_{a^{+}}^{\alpha} \psi^{L}(t)=f\left(t, \psi^{L}(t)\right)-g(t), \\
\psi^{L}(a)=\psi_{0}-\varepsilon_{1}, \quad\left(\psi^{L}\right)^{\prime}(a)=\xi_{0}-\varepsilon_{2},
\end{array}\right.
$$

where $t \in(a, b]$. Using Remark 4 and the definition (28), we have that

$$
\begin{aligned}
& \psi^{L}(t)=\left(\psi_{0}-\varepsilon_{1}\right) E_{\alpha, 1}\left(\lambda(\ln t-\ln a)^{\alpha}\right) \\
& +\psi^{(1)}\left(a, \varepsilon_{2}\right)(\ln t-\ln a) E_{\alpha, 2}\left(\lambda(\ln t-\ln a)^{\alpha}\right) \\
& +\int_{a}^{t} \frac{E_{\alpha, \alpha}\left(\lambda(\ln t-\ln s)^{\alpha}\right)}{1(\ln t-\ln s)^{1-\alpha}}\left[\begin{array}{l}
f\left(s, \psi^{L}(s)\right) \\
+\lambda \psi^{L}(s) \\
-g(s)
\end{array}\right] \frac{d s}{s} \\
& \leq\left(\mathbb{P} \psi^{L}\right)(t),
\end{aligned}
$$

where $\psi^{(1)}(a) \geq \psi^{(1)}\left(a, \varepsilon_{2}\right):=\lim _{t \rightarrow a}\left[t\left(\psi^{L}\right)^{\prime}(t)\right]$. Similarly, $\psi^{U} \geq \mathbb{P} \psi^{U}$ is satisfied. Set $\mathbb{D}:=$ $\left[\psi^{L}, \psi^{U}\right]$. Based on Step 2, we have that the operator $\mathbb{P}: \mathbb{D} \rightarrow C[a, b]$ is relatively compact, and so it follows that $\mathbb{P}$ is completely continuous. In addition, the condition (27) yields $f$ is monotone nondecreasing in $\psi$ if $M=0$. Thus, it follows that the operator $\mathbb{P}$ is nondecreasing on $\mathbb{D}$. Using Lemma 1 , the existence of the extremal solutions of the initial value problem (10) is obtained. The proof is complete.

As in the proof of Theorem 1, we also obtain the below corollary.

Corollary 1. Let $\alpha \in(1,2), f:[a, b] \times \mathbb{R} \rightarrow$ $\mathbb{R}$ be continuous and $\sup _{t \in[a, b]}|f(t, \psi)| \leqslant K_{f}$, where $K_{f}$ is a positive constant. Then, the 
problem (10) has at least one solution on $[a, b]$. Furthermore, if the Lipschitz condition given by (26) is satisfied, then the problem (10) has a unique solution on $[a, b]$.

\section{Stabilization of a fractional differential equation with order $\alpha \in(1,2)$}

In this section, we discuss the stability of the solution of the following problem

$$
\left\{\begin{array}{l}
C-H D_{a^{+}}^{\alpha} \psi(t)=A \psi(t)+g(t, \psi(t)) \\
\psi(a)=\psi_{0}, \quad \psi^{\prime}(a)=\psi^{\prime}
\end{array}\right.
$$

where $t \geq a \geq 1, \alpha \in(1,2), A$ is a constant, and the continuous and bounded function $g$ : $[a, b] \times \mathbb{R} \rightarrow \mathbb{R}$ is the nonlinear term of state $\psi(t)$ and it satisfies $g(t, 0) \equiv 0$. We observe that the state $\psi=0$ is the equilibrium of the problem (30), which can be taken as a target orbit. In this section, to force the state of the problem into the target orbit, we shall choose a linear feedback controller $u(t)=B \psi(t)$ to the problem (30). Therefore, we consider the following the controlled problem

$$
\left\{\begin{array}{l}
{ }^{C-H} D_{a^{+}}^{\alpha} \psi(t)=(A+B) \psi(t)+g(t, \psi(t)) \\
\psi(a)=\psi_{0}, \quad \psi^{\prime}(a)=\psi_{0}^{\prime}
\end{array}\right.
$$

where $t \geq a$, and the feedback gain $B$ needs to be determined, $\alpha \in(1,2)$.

Remark 7. Based on the result of Section 3, by putting $f(t, \psi)=A \psi(t)+g(t, \psi(t))$, then from the continuous hypothesis of the function $f$ we observe that the problem (30) has at least solution on $[a, b]$.

Theorem 2. We assume that for all $t \geq a$, $\alpha \in(1,2)$, the nonlinear term $g(t, z(t))$ satisfies the condition $\lim _{|z| \rightarrow 0} \frac{|g(t, z)|}{|z|}=0$. In addition, suppose that there exist $\rho>0$ and $\mu>0$ such that

$$
\begin{aligned}
& \left|E_{\alpha, \alpha}\left((A+B)\left(\ln \tau_{2}-\ln \tau_{1}\right)^{\alpha}\right)\right| \\
& \leqslant\left(\ln \tau_{2}-\ln \tau_{1}\right)^{1-\alpha} \rho e^{-\mu\left(\ln \tau_{2}-\ln \tau_{1}\right)},
\end{aligned}
$$

where $\tau_{2}>\tau_{1} \geq a$. Then, the solution of the problem (31) can be controlled to the target orbit $\psi \equiv 0$ provided that $\max \left\{\left|\psi_{0}\right|,\left|\psi_{0}^{\prime}\right|\right\}<\theta$, where $\theta$ is a positive constant.

Proof. From the result of Remark 4, one can obtain the following solution of system (31):

$$
\begin{aligned}
& \psi(t)=\psi_{0} E_{\alpha, 1}\left((A+B)(\ln t-\ln a)^{\alpha}\right) \\
& +\psi^{(1)}(a)(\ln t-\ln a) E_{\alpha, 2}\left((A+B)(\ln t-\ln a)^{\alpha}\right) \\
& +\int_{a}^{t} \frac{E_{\alpha, \alpha}\left((A+B)(\ln t-\ln s)^{\alpha}\right)}{(\ln t-\ln s)^{1-\alpha}} g(s, \psi(s)) \frac{d s}{s}
\end{aligned}
$$

Now, because $\lim _{|\psi| \rightarrow 0} \frac{|g(t, \psi)|}{|\psi|}=0$, we can see that for a given constant $\varepsilon>0$, there exists $\mathbb{M}=$ $\mathbb{M}(\varepsilon)>0$ such that if $|\psi| \leq \mathbb{M}, \forall t \geq a$, one has

$$
|g(t, \psi)| \leq \varepsilon|\psi|, \forall t \geq a
$$

Thus, from the existence result of the solution, if $\max \left\{\left|\psi_{0}\right|,\left|\psi^{(1)}(a)\right|\right\}<\mathbb{M}$, there exists $\delta=\delta(\mathbb{M})$ such that if $t \in[a, \delta)$, we have

$$
|\psi(t)|<\mathbb{M}
$$

Furthermore, we have for all $\alpha \in(1,2)$ and $i \in$ $\{0,1,2, \ldots, \infty\}$

$$
\begin{aligned}
\frac{\Gamma(\alpha i+\alpha)}{\Gamma(\alpha i+1)} & \leq \frac{\Gamma(\alpha i+\alpha+1)}{\Gamma(\alpha i+2)} \\
& =\frac{B(\alpha i+\alpha+1,1-\alpha)}{\Gamma(1-\alpha)} \\
& \leq \frac{B(\alpha+1,1-\alpha)}{\Gamma(1-\alpha)} \\
& =\Gamma(1+\alpha), \\
\frac{\Gamma(\alpha i+\alpha)}{\Gamma(\alpha i+2)} & =\frac{B(\alpha i+\alpha, 2-\alpha)}{\Gamma(2-\alpha)} \\
& \leq \frac{B(\alpha, 2-\alpha)}{\Gamma(2-\alpha)} \\
& =\Gamma(\alpha),
\end{aligned}
$$

where $B(\cdot, \cdot)$ is the Beta function. Therefore, using condition (32) we have the following estimate 
for all $\alpha \in(1,2)$ and $t>a$

$$
\begin{aligned}
& \left|E_{\alpha, 1}\left((A+B)(\ln t-\ln a)^{\alpha}\right)\right| \\
& \leq \Gamma(\alpha+1)\left|E_{\alpha, \alpha}\left((A+B)(\ln t-\ln a)^{\alpha}\right)\right| \\
& \leq(\ln t-\ln a)^{1-\alpha} \Gamma(\alpha+1) \rho e^{-\mu(\ln t-\ln a)}, \\
& \left|E_{\alpha, 2}\left((A+B)(\ln t-\ln a)^{\alpha}\right)\right| \\
& \leq \Gamma(\alpha)\left|E_{\alpha, \alpha}\left((A+B)(\ln t-\ln a)^{\alpha}\right)\right| \\
& \leq(\ln t-\ln a)^{1-\alpha} \Gamma(\alpha) \rho e^{-\mu(\ln t-\ln a)} .
\end{aligned}
$$

Then, substituting (32), (34) and (37) into (33), we obtain that for all $t \in[a, \delta)$

$$
\begin{aligned}
& |\psi(t)| \\
& \leq \rho \Gamma(\alpha) \frac{e^{-\mu(\ln (t / a))}}{(\ln (t / a))^{\alpha-1}} \\
& \cdot\left(2\left|\psi_{0}\right|+(\ln (t / a))\left|\psi^{(1)}(a)\right|\right) \\
& +\varepsilon \int_{a}^{t} \frac{\left|E_{\alpha, \alpha}\left((A+B)(\ln (t / s))^{\alpha}\right)\right|}{(\ln (t / s))^{1-\alpha}}|\psi(s)| \frac{d s}{s} \\
& \leq \rho \Gamma(\alpha) \frac{e^{-\mu(\ln (t / a))}}{(\ln (t / a))^{\alpha-1}} \\
& \cdot\left(2\left|\psi_{0}\right|+(\ln (t / a))\left|\psi^{(1)}(a)\right|\right) \\
& +\varepsilon \rho \int_{a}^{t} \exp (-\mu(\ln t-\ln s))|\psi(s)| \frac{d s}{s} \\
& :=q(t)+I(t),
\end{aligned}
$$

where

$$
\begin{aligned}
q(t): & =\rho \Gamma(\alpha) \frac{e^{-\mu \ln (t / a)}}{(\ln (t / a))^{\alpha-1}} \\
& \cdot\left(2\left|\psi_{0}\right|+\ln (t / a)\left|\psi^{(1)}(a)\right|\right) \\
I(t): & =\varepsilon \rho \int_{a}^{t} \exp (-\mu(\ln t-\ln s))|\psi(s)| \frac{d s}{s} .
\end{aligned}
$$

$I(t)$ with respect to $t$ and by (38), we get

$$
\begin{aligned}
\frac{d}{d t} I(t) & =\varepsilon \rho t^{-1}|\psi(t)| \\
& -\varepsilon \mu \rho t^{-1} \int_{a}^{t} \exp (-\mu(\ln t-\ln s))|\psi(s)| \frac{d s}{s} \\
& \leq \varepsilon \rho t^{-1} q(t)+(\varepsilon \rho-\mu) t^{-1} I(t),
\end{aligned}
$$

and by using Gronwall's inequality and since $a \geq$ 1 , we also get the estimate

$$
\begin{aligned}
& I(t) \leq \varepsilon \rho \int_{a}^{t} q(s) \exp ((\varepsilon \rho-\mu)(\ln t-\ln s)) \frac{d s}{s} \\
& \leq \varepsilon \rho \Gamma(\alpha)\left(2\left|\psi_{0}\right|+(\ln t-\ln a)^{2-\alpha}\left|\psi^{(1)}(a)\right|\right) \\
& \cdot e^{-\mu(\ln t-\ln a)} \int_{a}^{t} \exp (\varepsilon \rho(\ln t-\ln s)) \frac{d s}{s} \\
& =\Gamma(\alpha)\left(2\left|\psi_{0}\right|+(\ln t-\ln a)^{2-\alpha}\left|\psi^{(1)}(a)\right|\right) \\
& \cdot \exp ((\varepsilon \rho-\mu)(\ln t-\ln a)) \\
& -\Gamma(\alpha)\left(2\left|\psi_{0}\right|+(\ln t-\ln a)^{2-\alpha}\left|\psi^{(1)}(a)\right|\right) \\
& \cdot \exp (-\mu(\ln t-\ln a)) .
\end{aligned}
$$

Therefore, we get the following estimate on $[a, \delta)$ :

$$
\begin{gathered}
|\psi(t)| \leq \Gamma(\alpha)\left(2\left|\psi_{0}\right|+(\ln \delta-\ln a)^{2-\alpha}\left|\psi^{(1)}(a)\right|\right) \\
\cdot \exp ((\varepsilon \rho-\mu)(\ln t-\ln a))
\end{gathered}
$$

Putting $\left|\hat{\psi}_{0}\right|:=\max \left\{\left|\psi_{0}\right|,\left|\psi^{(1)}(a)\right|\right\}$, and if $\varepsilon<$ $\frac{\mu}{\rho}$, then we get

$$
\begin{aligned}
|\psi(t)| & \leq \Gamma(\alpha)\left(2+(\ln \delta-\ln a)^{2-\alpha}\right)\left|\hat{\psi}_{0}\right| \\
& :=\mathbb{K}\left|\hat{\psi}_{0}\right|, \quad t \in[a, \delta) .
\end{aligned}
$$

This shows that if we choose $\theta=\min \left\{\mathbb{M}, \frac{\mathbb{M}}{2 \mathbb{K}}\right\}$, where $\mathbb{M}$ is defined by (35), then we obtain the following assertion: if $\left|\hat{\psi}_{0}\right|<\theta$ on $[a, \delta)$, then from (40) we have

$$
|\psi(t)|<\mathbb{K} \theta \leq \frac{\mathbb{M}}{2}, \quad t \in[a, \delta) .
$$

On the other hand, using Leibniz's rule for differentiation under the integral sign of the function
Next, we observe that the inequality (41) is also true if the value of $\delta$ is further extended. Indeed, 
consider the continuous solution $\psi(t)$ of system (31) such that $\left|\hat{\psi}_{0}\right|<\theta$ and $\varepsilon<\frac{\mu}{\rho}$, and let

$\mathbb{T}=\sup \left\{\delta_{1} \mid \delta_{1} \geq a\right.$ and if $t \in\left[a, \delta_{1}\right)$,

the solution $\psi(t)$ is defined and $|\psi(t)|<\mathbb{M}\}$.

Suppose that $\mathbb{T}$ is finite. Then, from (41) we have that $|\psi(t)|<\frac{\mathbb{M}}{2}$ on $[a, \mathbb{T})$. According to the existence theory in Section 3, it follows that the solution $\psi(t)$ can be further extended so that $\mathbb{T}$ is contained in the maximal existent interval $\left[a, \mathbb{T}^{*}\right)$, where $\mathbb{T}^{*}>\mathbb{T}$. Then, since $\psi(t)$ is continuous, one has $|\psi(t)|<\mathbb{M}$ on $\left[a, \mathbb{T}^{*}\right)$. Therefore, this leads to the contradiction of the definition of $\mathbb{T}$. Thus we conclude that if $\left|\hat{\psi}_{0}\right|<\theta$, then $|\psi(t)|<\mathbb{M}$ for all $t \geq a$. It further follows that the inequality (39) is valid for all $t \geq a$ and $\lim _{t \rightarrow \infty}|\psi(t)|=0$ provided that $\varepsilon<\frac{\mu}{\rho}$. This implies that the solution $\psi(t)$ of system (31) can be forced to the equilibrium $\psi \equiv 0$. The proof is complete.

Based on the assertion of Theorem 2, we get the following corollary.

Corollary 2. Assume that for all $t \geq a, \alpha \in$ $(1,2)$, the function $g(t, \psi)$ satisfies the condition $\lim _{|\psi| \rightarrow 0} \frac{|g(t, \psi)|}{|\psi|}=0$. In addition, suppose that there exist $\rho>0$ and $\mu>0$ such that

$$
\begin{aligned}
& \left|E_{\alpha, \alpha}\left((A+B)\left(\ln \tau_{2}-\ln \tau_{1}\right)^{\alpha}\right)\right| \\
& \leqslant\left(\ln \tau_{2}-\ln \tau_{1}\right)^{1-\alpha} \rho e^{-\mu\left(\ln \tau_{2}-\ln \tau_{1}\right)},
\end{aligned}
$$

where $\tau_{2}>\tau_{1} \geq a$. Then, there exists $\theta>0$ such that the equilibrium state $\psi \equiv 0$ of the problem (30) is asymptotically stable provided that $\max \left\{|\psi|,\left|\psi_{0}^{\prime}\right|\right\}<\theta$.

The following example is given to illustrate the assertion of Theorem 2.

Example 4. Let $[a, b]=[1,10], \alpha \in(1,2)$, $A=-1, \psi_{0}=\psi_{0}^{\prime}=1$, and $g(t, \psi):=$ $-(1 / 2) \cos (\psi(t)) \psi^{2}(t)$. We consider the following problem

$$
\left\{\begin{array}{l}
{ }^{C-H} D_{1+}^{\alpha} \psi(t)=-\psi(t)-\frac{1}{2} \cos (\psi(t)) \psi^{2}(t) \\
\psi(1)=1, \quad \psi^{\prime}(1)=1
\end{array}\right.
$$

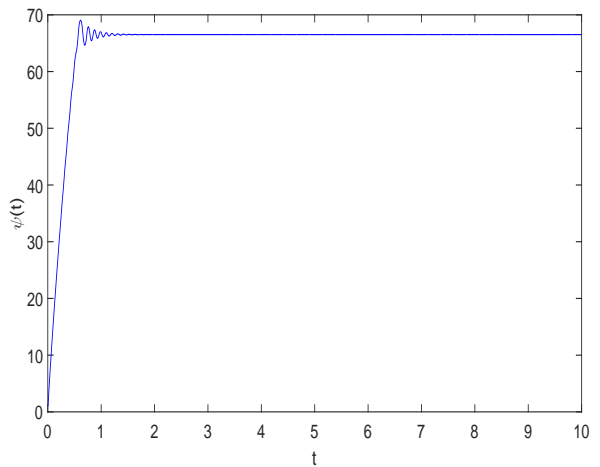

Fig. 8: The unstable orbit at the equilibrium point $\psi(t)=0$ of the solution of the problem (43).

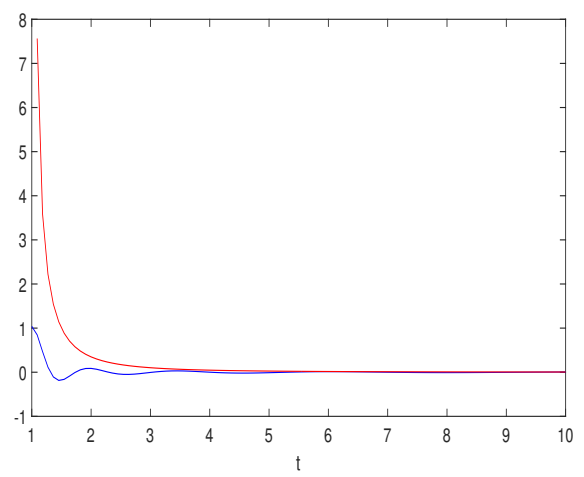

Fig. 9: The graph of the condition (32) with the lefthand side (blue) and the right-hand side (red).

In this example, if we take $\alpha=1.9$, then problem (43) is unstable at the equilibrium point $\psi(t)=0$. The unstable orbit of the problem (43) is numerically shown in Fig. 8. Now, we assume that the equilibrium point 0 is the target orbit. To force the trajectory of the problem (43) into the target orbit, we shall add a feedback controller $u(t)=B \psi(t)$ to the problem (43), where the constant $B$ is a control gain. Based on Theorem 2, if the control gain is taken as $B=100$ and the parameters are chosen $\varepsilon=1, \theta=1, \rho=1.5$, $\mu=2$, then the condition (32) is valid for the above-mentioned control gain and parameters (see Fig. 9). According to Theorem 2, this implies that the orbit of the problem (43) can be controlled to equilibrium $\psi(t)=0$ via the feedback controller $u(t)=B \psi(t)$. The graph of the controlled orbit of the problem (43) is shown in Fig. 10. 


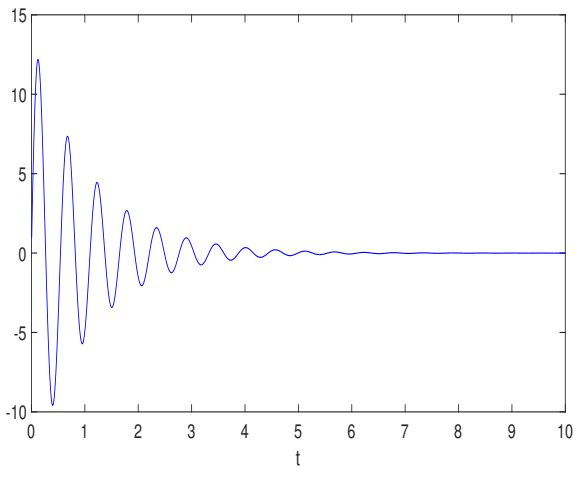

Fig. 10: The graph of the controlled orbit of the problem (43).

\section{Conclusion}

In the paper, by using the well-known method of lower and upper solutions, the existence theory of the extremal solutions for a class of fractional differential equations under the CaputoHadamard derivative with the case of $\alpha \in(1,2)$ has been established. We also provide sufficient conditions that ensure the stability of a class of fractional differential equations. Finally, an example was implemented to demonstrate the feasibility and validity of the proposed method, which were consistent with the theoretical results. The approach proposed in this paper may be extended to other fractional differential systems and in the future, it will be applied to investigate the stabilization of non-linear systems with the fractional derivative.

\section{Acknowledgments}

The authors are very grateful to the referees for their valuable suggestions, which helped to improve the paper significantly. The authors would like to thank the Vietnam National Foundation for Science and Technology Development (NAFOSTED) under grant number 107.02-2017.319.

\section{References}

[1] Adjabi, Y., Jarad, F., Baleanu, D., \& Abdeljawad, T. (2016). On Cauchy problems with Caputo Hadamard fractional derivatives. J. Comput. Anal. Appl, 21(4), 661681.

[2] Almeida, R. (2017). Caputo-Hadamard fractional derivatives of variable order. $\mathrm{Nu}^{-}$ merical Functional Analysis and Optimization, 38(1), 1-19.

[3] Bai, Z., Zhang, S., Sun, S., \& Yin, C. (2016). Monotone iterative method for fractional differential equations. Electron. J. Differ. Equ, 6, 2016.

[4] Gambo, Y. Y., Jarad, F., Baleanu, D., \& Abdeljawad, T. (2014). On Caputo modification of the Hadamard fractional derivatives. Advances in Difference Equations, 2014(1), 10.

[5] Jarad, F., Abdeljawad, T., \& Baleanu, D. (2012). Caputo-type modification of the Hadamard fractional derivatives. Advances in Difference Equations, 2012(1), 142.

[6] Guo, D., Sun, J., \& Liu, Z. (1995). Functional Methods for Nonlinear Ordinary Differential Equations. Shandong Science and Technology Press, Jinan, China.

[7] Kilbas, A. A., Srivastava, H. M., \& Trujillo, J. J. (2006). Theory and applications of fractional differential equations (Vol. 204). elsevier.

[8] Klimek, M. (2011). Sequential fractional differential equations with Hadamard derivative. Communications in Nonlinear Science and Numerical Simulation, 16(12), 4689-4697.

[9] McRae, F. A. (2009). Monotone iterative technique and existence results for fractional differential equations. Nonlinear Analysis: Theory, Methods \& Applications, 71(12), 6093-6096.

[10] Pei, K., Wang, G., \& Sun, Y. (2017). Successive iterations and positive extremal solutions for a Hadamard type fractional 
integro-differential equations on infinite domain. Applied Mathematics and Computation, 312, 158-168.

[11] Wei, Z., Li, Q., \& Che, J. (2010). Initial value problems for fractional differential equations involving Riemann-Liouville sequential fractional derivative. Journal of Mathematical Analysis and Applications, $367(1), 260-272$.

[12] Zhang, W., Bai, Z., \& Sun, S. (2016). Extremal solutions for some periodic fractional differential equations. Advances in Difference Equations, 2016(1), 179.

[13] Yukunthorn, W., Ahmad, B., Ntouyas, S. K., \& Tariboon, J. (2016). On Caputo-Hadamard type fractional impulsive hybrid systems with nonlinear fractional integral conditions. Nonlinear Analysis: Hybrid Systems, 19, 77-92.

[14] Zhang, S. (2009). Monotone iterative method for initial value problem involving Riemann-Liouville fractional derivatives. Nonlinear Analysis: Theory, Methods \& Applications, 71(5-6), 2087-2093.
[15] Wang, G., Baleanu, D., \& Zhang, L. (2012). Monotone iterative method for a class of nonlinear fractional differential equations. Fractional Calculus and Applied Analysis, 15(2), 244-252.

\section{About Authors}

Donal O'REGAN is a Professor of Mathematics at the National university of Ireland, Galway, Ireland. His research interest is in nonlinear functional analysis and has written many papers and books in this area.

Van Hoa NGO is currently an researcher at Institute for Computaional Science, Ton Duc Thang University, Ho Chi Minh City, Vietnam. He received his B.Sc degree in Mathematics and Computer Science from University of Science-Vietnam National University in 2010. $\mathrm{He}$ is currently studying for the Ph.D. program in Applied Mathematics at University of Science-Vietnam National University. His research interests include Uncertain differential equations; Fractional differential equations; Stability of fractional dynamical systems; Dynamical control systems. 